\title{
Feeling present in arousing virtual reality worlds: prefrontal brain regions differentially orchestrate presence experience in adults and children
}

\author{
Thomas Baumgartner,2,*, Dominique Speck ${ }^{1}$, Denise Wettstein ${ }^{1}$, Ornella Masnari', \\ Gian Beeli' and Lutz Jäncke' \\ 1. Institute of Psychology, Department of Neuropsychology, University of Zurich, Switzerland \\ 2. Institute for Empirical Research in Economics, Center for the Study of Social and Neural Systems, University of Zurich, Switzerland \\ Edited by: Patrik Vuilleumier, University Medical Center and University Hospital Geneva, Switzerland \\ Reviewed by: Lars Schwabe, Brain Mind Institute, EPFL, Lausanne, Switzerland \\ Thomas Parsons, Institute for Creative Technologies, University of Southern California, USA
}

\begin{abstract}
Virtual reality (VR) is a powerful tool for simulating aspects of the real world. The success of VR is thought to depend on its ability to evoke a sense of "being there", that is, the feeling of "Presence". In view of the rapid progress in the development of increasingly more sophisticated virtual environments (VE), the importance of understanding the neural underpinnings of presence is growing. To date however, the neural correlates of this phenomenon have received very scant attention. An fMRI-based study with 52 adults and 25 children was therefore conducted using a highly immersive VE. The experience of presence in adult subjects was found to be modulated by two major strategies involving two homologous prefrontal brain structures. Whereas the right DLPFC controlled the sense of presence by down-regulating the activation in the egocentric dorsal visual processing stream, the left DLPFC up-regulated widespread areas of the medial prefrontal cortex known to be involved in self-reflective and stimulus-independent thoughts. In contrast, there was no evidence of these two strategies in children. In fact, anatomical analyses showed that these two prefrontal areas have not yet reached full maturity in children. Taken together, this study presents the first findings that show activation of a highly specific neural network orchestrating the experience of presence in adult subjects, and that the absence of activity in this neural network might contribute to the generally increased susceptibility of children for the experience of presence in VEs.
\end{abstract}

Keywords: virtual reality, presence experience, prefrontal cortex, cognitive/executive control, brain maturation, adults, children

\section{INTRODUCTION}

Virtual reality (VR) is being used in recreation, entertainment, education, psychotherapy, and medicine as a tool for simulating aspects of the real world (Klinger et al., 2005, 2006; Parsons and Rizzo, 2008; Parsons et al., 2007; Slater et al., 2006). The success of VR is thought to be associated with the subjective sense of "being there" that VR can evoke (Steuer et al., 1995). This feeling is widely referred to as presence (Sanchez-Vives and Slater 2005). Presence may be understood as the subjective experience of being transiently unaware of actions and cognitions linked to the real environment while concurrently perceiving oneself as situated in and perceiving action possibilities associated with the virtual environment (VE) (Wirth et al., 2007). Or in other words, presence is defined as an egocentric spatial experience of VEs. Interestingly, there are differences in the extent

*Correspondence: Thomas Baumgartner, Institute for Empirical Research in Economics, Center for the Study of Social and Neural Systems, University of Zürich, Blümlisalpstrasse 10, CH-8006 Zürich, Switzerland. e-mail: t.baumgartner@iew.uzh.ch

Received: 16 May 2008; paper pending published: 02 July 2008; accepted: 06 August 2008; published online: 25 August 2008.

Citation: Front. Hum. Neurosci. (2008) 2: 8. doi: 10.3389/neuro.09.008.2008

Copyright $\odot 2008$ Baumgartner, Speck, Wettstein, Masnari, Beeli and Jäncke. This is an openaccess article subject to an exclusive license agreement between the authors and the Frontiers Research Foundation, which permits unrestricted use, distribution, and reproduction in any medium, provided the original authors and source are credited. to which individuals experience presence while for example playing a video game or watching a film. Some individuals are easily drawn into the virtual environment while others do so more slowly or indeed fail to develop any sense of presence at all (Ijsselsteijn and Riva, 2003; Wirth et al., 2007). Furthermore, there is an ongoing discussion as to whether children and adults actually differ in the quality and speed with which presence is evoked by VEs (Schaik et al., 2004). In view of the rapid progress in the development of increasingly more sophisticated virtual environments, the importance of understanding the neural underpinnings of presence and the brain activity associated with inter-individual differences in the experience of presence (1) in adults, (2) in children and in particular (3) between adults and children is growing.

A structural candidate in the brain which might be involved in modulating the inter-individual differences in the experience of presence is the prefrontal cortex - an important area of the brain that houses most of the so-called executive functions that exert regulatory and in particular inhibitory control over emotions and our behaviour (Aron et al., 2004; Garavan et al., 1999; Knoch et al., 2006a,b; Koechlin and Hyafil, 2007; Koechlin et al., 2003; Ridderinkhof et al., 2004). Moreover, the brain undergoes significant maturational changes during the course of development to adulthood, the prefrontal cortex (PFC) taking particularly long to reach full maturity (Sowell et al., 1999). 
Recent longitudinal studies in children and adolescents have uncovered extensive anatomical changes in the frontal cortex until early adulthood (Giedd, 2004; Giedd et al., 1999; Gogtay et al., 2004; for a review see Toga et al., 2006). Thus, it is conceivable that children are more strongly "drawn" into the world presented in VR because they experience greater difficulty in inhibiting the feeling of presence, and that differences between adults and children in the experience of presence in a VE are associated in some way with corresponding maturational and related functional differences in the PFC.

As presence is understood as an egocentric spatial experience of VEs, it is also very likely that parietal brain regions (including in particular regions of the dorsal visual stream) play an essential role in this experience because these brain structures are involved in generating an egocentric view by translation of the retinal coordinates to head-centered, or even body-centered coordinates (Gron et al., 2000; Jordan et al., 2004). Moreover, these parietal regions are strongly anatomically connected with dorsal structures of the prefrontal cortex (Croxson et al., 2005) and thus, we speculate that inter-individual differences in activation of the parietal cortex and associated presence experience might be modulated by prefrontal brain structures.
In order to investigate the proposed impact of the prefrontal cortex on parietal regions and associated presence experience in the context of maturational differences, we conducted an fMRIbased study on a large sample of healthy adults $(n=52 ; 26$ female; mean age $=26.2$, S.D. $=4.1)$ and children $(n=25 ; 11$ female; mean age $=8.7$, S.D. $=1.6)$ exposed to two different variants of a virtual roller coaster scenario (Figure 1A). One scenario consisted of realistic roller coaster rides with spectacular ascending and descending sections and loops (referred to as High Presence condition), whereas in the other scenario the roller coaster cart followed the bending but horizontal path of the tracks (referred to as Low Presence condition). A recent study (Baumgartner et al., 2006b) has shown that these two scenarios evoke different presence experiences (both in terms of quality and intensity) as measured with a standard presence rating questionnaire (Vorderer et al., 2004). We used the same presence questionnaire in the current study, with a large subject pool in order to obtain strong variability in presence scores. These scores were then used in different brain imaging analyses, as regressors in across-subjects correlational analyses and grouping variables in within-subjects effective connectivity analyses (see below), in order to reveal the nature and extent of inter-individual differences in cerebral
A

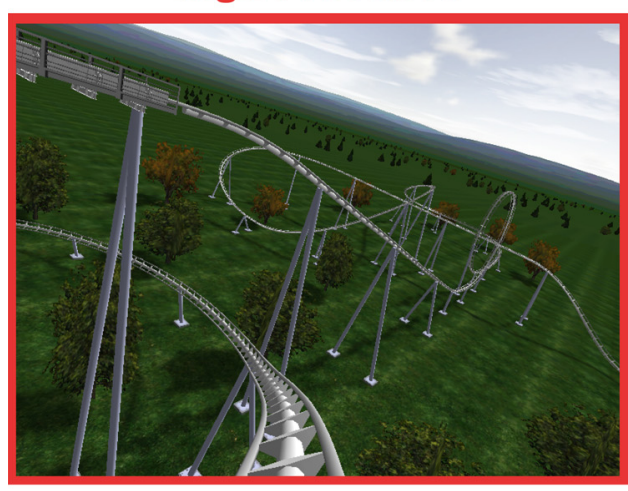

B

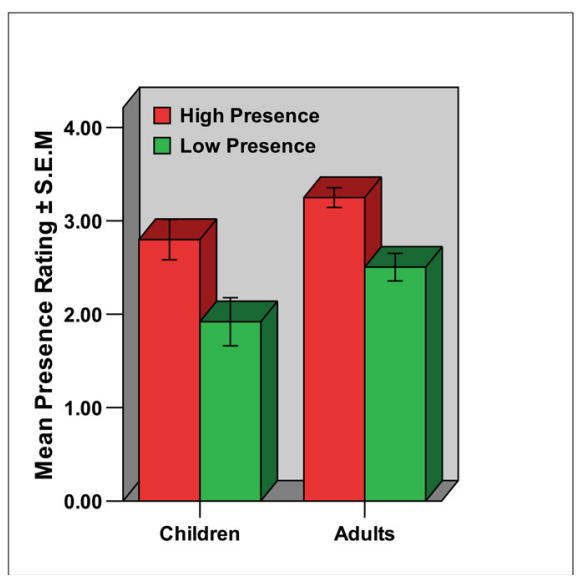

Low Presence

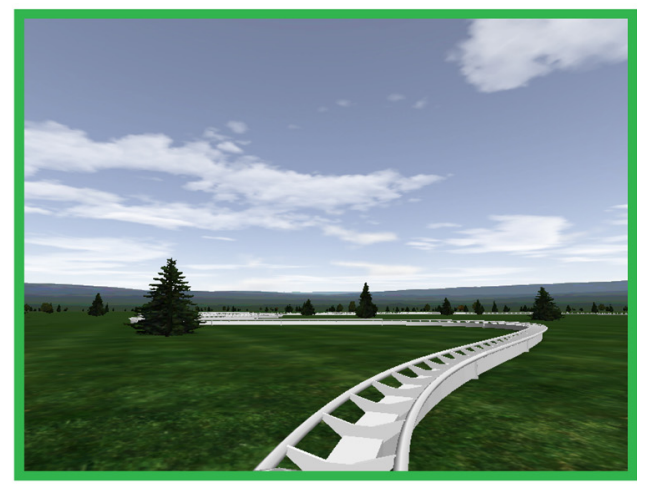

C

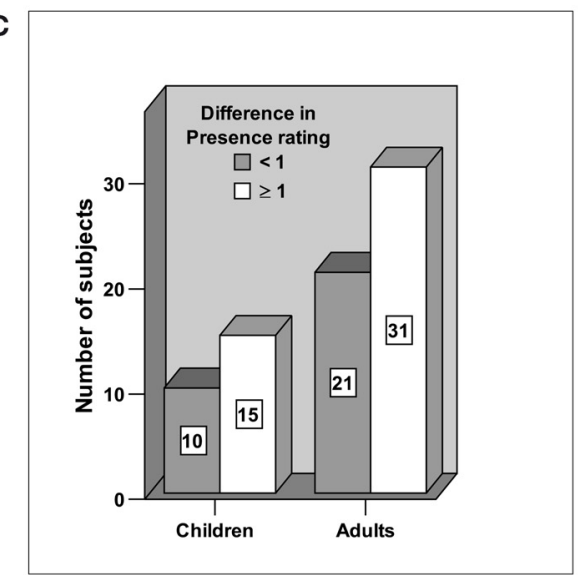

Figure 1 | Experimental design and Presence rating. (A) Experimental paradigm. Different roller coaster scenarios were presented by means of MR-compatible goggles and earphones. Whereas in the High Presence condition the roller coaster ride consisted of spectacular ascending and descending sections and loops, in the Low Presence condition the roller coaster cart followed a winding but horizontal path. (B) Depicted is the mean \pm S.E.M. in Presence rating on a 5-point scale, broken down for group (adults/children) and condition (High Presence/Low Presence). The scale measures the subjective impression of how much the subjects felt they were situated in the midst of the action of the roller coaster ride rather than merely observing it. Both adults and children clearly indicated an enhanced presence experience in the High compared to the Low Presence condition. (C) Similar distribution of presence ratings in adults and children, indicating that about $40 \%$ of subjects in each group reported a difference in presence rating of less than one between the High and Low Presence condition (referred to in the paper as low Presence rating group), whereas about $60 \%$ of subjects in each group reported a difference in the presence rating of higher than or equal to one (referred to as high Presence rating group). 
networks orchestrating the presence experience (1) in adults, (2) in children, and in particular (3) between adults and children.

\section{MATERIALS AND METHODS SUBJECTS}

Fifty-two $(n=52)$ healthy right-handed adults ( 26 female; mean age $=26.2$, S.D. $=4.1$, range: $21-43)$ and twenty-five $(n=25)$ healthy right-handed children (11 female, mean age $=8.7$, S.D. $=1.6$, range: 6-11) participated in the fMRI experiment. Handedness was determined with a standard rating scale (Annett, 1970). No adult subjects, but four children were excluded from the functional analysis due to excessive motion $(>2 \mathrm{~mm})$. After having eliminated these subjects from the analyses, the two age groups did not differ significantly in in-plane motion. For the analyses of the anatomical data, we had to excluded four children and three adults due to distorted anatomical images based on visual inspection. Moreover, due to technical reasons, we only obtained in 40 of the 52 adult subjects anatomical images. Subjects had no history of neurological or psychiatric disorder and each gave informed consent (parental consent and child assent for children) for a protocol approved by the institutional ethics committee of the University of Zurich. The study was carried out in accordance with the Declaration of Helsinki principles and subjects were informed of their right to discontinue participation at any time.

\section{DESIGN: VIRTUAL ENVIRONMENT}

The subjects were given the illusion of sitting in the front cart of a roller coaster while watching different scenarios. These environments were created with the software "NoLimits Roller Coaster Simulation" (www.nolimitscoaster.com), embedded in "Presentation" (Neurobehavioral System, Version 10.0) and presented by means of MR-compatible goggles (VisuaStim XGA; Resonance Technology, Northridge, California, $800 \times 600$ resolution at $60 \mathrm{~Hz}$; visual field $=30^{\circ} \times 24^{\circ}$; maximum contrast ratio $=18: 1)$ and headphones.

The experimental condition referred to as High Presence condition (Figure 1A, left) consisted of "realistic" roller coaster rides, divided into three different phases: anticipation, dynamic and end. In the anticipation phase, subjects viewed tracks leading upwards and heard a pulling sound (as if their cart was being mechanically pulled up) until reaching the peak of the anticipation phase where the dynamic phase began. In this dynamic phase, the roller coaster ride consisted of spectacular ups, downs and loops in all three dimensions of space and different acceleration und brake passages. The sound of the dynamic phase supported the visual input by different intensities of driving noise with spatial cues. At the end of each ride the cart braked (with braking noise) to lower velocity on a straight track which is referred to as end phase. The names of the roller coasters used in the present study are as follows: Giant, Revolution, Silverstar, Viper. They can be inspected using the mentioned software "NoLimits Roller Coaster Simulation" (www.nolimitscoaster.com).

The control condition referred to as Low Presence condition (Figure 1A, right) consisted of horizontal straight tracks in the anticipation and end phase and sinuous-lined tracks with different velocity in the dynamic phase in an equivalent visual and acoustic (driving noise with spatial cues) environment. This control scenario was used in order to control for various basic perceptual processing steps similar for all roller coaster scenarios, including form, color, motion, and basic spatial processing.
We have chosen these two experimental conditions because various behavioral studies have shown that the number of spatial cues, both visual and auditory, are positively correlated with the Presence experience in the observers (Freeman et al., 1999; Ijsselsteijn et al., 1998, 2001). In our paradigm, monocular visual and auditory spatial cues are increased in the High Presence compared to the Low Presence condition primarily in the dynamic phase of the roller coaster ride. Thus, we focused our analyses in this paper on the dynamic phase. Results of the anticipation phase will be reported elsewhere.

Each ride lasted $102 \mathrm{~s}$ in total, whereas the different phases where divided into the following time scheme: anticipation phase $30 \mathrm{~s}$, dynamic phase $60 \mathrm{~s}$ and end phase $12 \mathrm{~s}$. In total, eight different roller coaster rides were presented, four High Presence and four Low Presence roller coaster rides. The different rides were separated by a fixation baseline which lasted $21 \mathrm{~s}$. Thus, the paradigm had a length of $16 \mathrm{~min}$ and $24 \mathrm{~s}$. In order to ensure that all roller coasters of the High Presence condition fitted to the mentioned time scheme for the different phases of the ride, they had to be slightly adapted at the beginning and end of the ride.

\section{POST-SCAN QUESTIONNAIRES}

After scanning, subjects completed a post-scan questionnaire which asked them to indicate on a 5-point analogue Likert scale their subjective Presence experience during the High and Low Presence conditions. This questionnaire scale measures the subjective impression how much they felt to be in the middle of the action of the roller coaster ride rather than merely observing it (Vorderer et al., 2004; Wirth et al., 2007). In order to assess subjective arousal and valence, we used the Self Assessment Manikin (SAM) (Lang, 1985). SAM depicts each dimension with a graphic character arrayed along a continuous 5-point scale. For pleasure, SAM ranges from a smiling, happy figure to a frowning, unhappy figure; for arousal SAM ranges from sleepy with eyes closed to excited with eyes open. The questions were carefully explained to the adults, but in particular to the children in order to ensure that they were able to understand and answer the questions properly.

\section{fMRI IMAGE ACQUISITION AND ANALYSES \\ Image acquisition and preprocessing}

Functional MRI acquisition was performed on a $3 \mathrm{~T}$ wholebody scanner (GE Medical, Milwaukee, WI, USA), using gradient-recalled echo planar imaging (repetition time, $\mathrm{TR}=3 \mathrm{~s}$; echo time, $\mathrm{TE}=32 \mathrm{~ms}$, flip angle $=90^{\circ}$, field of view $($ FOV $)=240 \mathrm{~mm} \times 240 \mathrm{~mm}$; matrix size $=64 \times 64$; voxel size $=$ $3.91 \mathrm{~mm} \times 3.91 \mathrm{~mm}, 30$ contiguous slices parallel to the AC-PC line, slice thickness $=3.9 \mathrm{~mm}$ ). For the preprocessing and statistical analyses, the statistical parametric mapping software package (SPM5, Wellcome Department of Cognitive Neurology, London, UK) implemented in Matlab (Version 7) were used. For functional analysis, all images were realigned to the first volume, corrected for motion artefacts and normalized $\left(3 \times 3 \times 3 \mathrm{~mm}^{3}\right)$ into standard stereotaxic space (template provided by the Montreal Neurological Institute). We used the same template for adults and children because previous methodological work has shown that the stereotactic normalization and time course of the hemodynamic response across the ages tested in the current study are not dissimilar (Burgund et al., 2002; Kang et al., 2003). After normalization, data were smoothed using an 8-mm full-width-at-half-maximum Gaussian kernel. A band-pass filter, which was composed of a discrete cosine-basis function with 
a cut-off period of $196 \mathrm{~s}$ for the high-pass filter was applied. In order to increase signal to noise ratio, global intensity changes were minimized by scaling each image to the grand mean.

\section{Random-effects analyses}

We performed random-effects analyses on the functional data for the dynamic phase of the roller coaster ride. For that purpose, we defined a general linear model (GLM) that included the following six regressors: High Presence anticipation, High Presence dynamic and High Presence end as well as Low Presence anticipation, Low Presence dynamic and Low Presence end. All regressors were convolved with a canonical haemodynamic response function (HRF) and the durations of the regressors were modeled according to the duration of the different phases of the roller coaster ride: anticipation phase $=30 \mathrm{~s}$, dynamic phase $=60 \mathrm{~s}$ and end phase $=12 \mathrm{~s}$. The six scan-to-scan motion parameters produced during realignment were included as additional regressors in the SPM analysis to account for residual effects of scan to scan motion. The correction for multiple comparison in wholebrain analyses was carried out using an uncorrected $p$ value of either 0.001 or 0.005 combined with a cluster-size threshold of 10 voxels (Forman et al., 1995).

In a first random effects group analyses, we calculated separately for adults and children the contrast High Presence > Low Presence in the dynamic phase of the roller coaster ride, revealing strong activations for both groups mainly in the dorsal and ventral visual processing stream (at $p<0.001$, cluster extent: 10 voxels). In addition, we used the following two serial subtraction terms thresholded at $p<0.005$ (cluster extent: 10 voxels) to uncover age group differences in the dynamic phase of the roller coaster ride: Adult subjects (High Presence $>$ Low Presence) $>$ Children subjects (High Presence $>$ Low Presence) and vice versa. The serial subtraction terms were masked inclusively with the first contrast of the serial subtraction term at $p<0.005$.

In a second random effects group analyses, we used the individual Presence rating differences between the High Presence and Low Presence condition as a covariate to identify brain regions in the prefrontal cortex (based on our hypotheses) that correlated negatively with the Presence rating differences in the brain contrast High Presence $>$ Low Presence. This analysis, again separately conduced for adults and children, revealed only in adult subjects two homologues areas of the DLPFC which correlated negatively with the difference in Presence rating (at $p<0.001$ in the right hemisphere and at $p<0.005$ in the left hemisphere, cluster extent: 10 voxels).

\section{Psychophysiological interaction analyses (PPI)}

To assess task-dependent changes in effective connectivity (positive or negative) between bilateral DLPFC and other brain regions, we performed Psychophysiological Interaction analyses (PPI) (Friston et al., 1997). Individual mean-corrected time series of the left and right DLPFC were extracted from functional ROIs based on the correlational analyses described above. Two separate analyses, one for the left and one for the right DLPFC, were conducted both for adults and children. The PPI was defined as the element-by-element product (interaction term) of the respective DLPFC time series and a vector coding for the two main effects High Presence and Low Presence (by subtracting High Presence-Low Presence in the dynamic phase).

For second-level random effects PPI analysis, the single-subject contrasts were entered into two three-way ANOVAs conducted separately for negative and positive connectivity. The factors in these two three-way ANOVAs were as follows: "age group" (adults/ children) and "rating group" (high Presence/low Presence rating group) as between-subject factors and "lateralization" (left/right DLPFC) as a within-subject factor. Importantly, we only sought for negative connectivity with right or left DLPFC in brain regions activated in the contrast High Presence $>$ Low Presence calculated in adult subjects and thresholded at $p<0.001$ with a cluster extent of 10 voxels (Figure S1 and Table S2). As shown in Figure S1, adults and children demonstrated in this contrast a highly similar brain activation pattern. However, the extent of activation was slightly increased in adult subjects. Thus, in order to search for negative connectivity in the same brain regions both in adults and children, we masked the negative connectivity analyses with the results of the adult group contrast. Similarly, we only searched for positive connectivity in brain regions not activated in the contrast High Presence $>$ Low Presence calculated in adult subjects and thresholded at $p<0.001$ with a cluster extent of 10 voxels. With regard to the positive connectivity analyses, we focused our analyses mainly on medial prefrontal cortex structures, including anterior cingulate cortex. Please see the 'Results' section for the reasons of these masking procedures.

Based on the two described PPI ANOVAs, we first calculated, separately for adults and children and irrespective of the Presence rating, the negative and positive connectivity with both the right and left DLPFC at $p<0.005$ with a cluster extent of 10 voxels. Significant results indicate a stronger negative or positive connectivity in the High Presence condition compared to the Low Presence condition. In regions showing such a negative or positive connectivity effect either with the right or the left DLPFC, we next tested for statistical significant lateralization and age group effects. On the basis of a strongly diminished search volume, we report differential lateralization and age group results in these regions at $p<0.005, p<0.01$ and $p<0.05$ (all uncorrected) with a cluster extent of 10 voxels. The conjunctive probability of two such events (i.e., a cluster being significant in both of these analyses) is for the lowest significance level $p<0.00025$ (i.e., $0.005 \times 0.05$ ), uncorrected for multiple comparisons, and is thus more conservative than the $p<0.001$, uncorrected threshold that is commonly used in fMRI studies. Highly similar approaches have been used in recent publications (Eisenberger et al., 2007; Harbaugh et al., 2007; Thielscher and Pessoa, 2007) in order to solve the multiple comparison problems in neuroimaging studies. In addition, we calculated for all regions showing significant age group effects small volume family-wise-error (FWE) corrections based on spherical ROIs ( $5 \mathrm{~mm}$ radius) build around the peak of activation.

Finally, we examined, separately for adults and children subjects, whether subjects of the Low Presence rating group compared to subjects of the High Presence rating group demonstrated differences in negative or positive connectivity with right and left DLPFC, at $p<0.005$ with a cluster extent of 10 voxels. For those regions demonstrating a differential rating group effect, we created spherical ROIs of $5 \mathrm{~mm}$ radius using the MarsBaR software. Using these ROIs and the software package SPSS (Version 13), we conducted repeated-measures ANOVAs and independent $t$-tests using participants' mean beta weights to further investigate lateralization patterns (left/right DLPFC), age group (adults/children) and rating group (High Presence/Low Presence rating group) effects.

\section{ANATOMICAL IMAGE ACQUISITION AND ANALYSES}

Structural image acquisition consisted of 172 T1-weighted transversal images (matrix size $=256 \times 256$; voxel size $=0.9375 \mathrm{~mm} \times$ $0.9375 \mathrm{~mm}$; slice thickness $1 \mathrm{~mm}$ ). For anatomical analyses, we 
used voxel-based morphometry (VBM) to investigate whether the two homologues DLPFC brain regions identified in the correlational analyses and used for the PPI analyses as seed regions, show, as expected, age group differences in gray matter density and gray matter volume. VBM is a whole-brain technique that is capable of discovering subtle, regionally specific changes in gray matter by averaging across subjects. This method is based on high-resolution structural three-dimensional magnetic resonance images, registered in standard space, and is designed to find significant regional differences throughout the brain by applying voxelwise statistics within the context of Gaussian random fields (Ashburner and Friston, 2000).

The data preprocessing and analysis were performed with VBM5 implemented in SPM5 (Wellcome Department of Cognitive Neurology, London, UK). Preprocessing of the data involved spatial normalization, segmentation, modulation, and spatial smoothing with a Gaussian kernel (Ashburner and Friston, 2000, 2005). In addition, we also analysed the anatomical images without the modulation option which involves scaling by the amount of contraction, so that the total amount of gray matter in the modulated GM remains the same as it would be in the original images. We follow in the paper the commonly used terms "volume" for modulated data and "density" (or concentration) for unmodulated data, respectively.

\section{RESULTS}

\section{PSYCHOMETRICAL RESULTS}

The psychometric scores indicated that, as expected, adults and children demonstrated an increase in their ratings of presence (Figure 1B), arousal, and valence in the High Presence condition compared with the Low Presence condition (paired $t$-tests: all $p<0.01$, Table S1). Importantly, the extent of the difference in the presence rating between the High Presence and the Low Presence condition was similar in adults and children (independent $t$-test: $t=0.56, \mathrm{df}=75, p=0.574)$. And, an equal number of adults and children reported a difference in their presence rating of less than 1 (about $40 \%$ ) or greater than (or equal to) 1 (about 60\%), thus demonstrating a comparable distribution of presence ratings in adults and children (Figure 1C). However, the difference rating for arousal was enhanced in adults (independent $t$-test: $t=3.588, \mathrm{df}=75, p<0.01$ ), and the difference rating for valence was increased in children (independent $t$-test: $t=3.057, \mathrm{df}=75, p<0.01)$.

\section{NEUROIMAGING RESULTS \\ High Presence $>$ Low Presence}

We first analyzed the functional magnetic resonance imaging (fMRI) data separately for each age group for the contrast High Presence > Low Presence. As discussed in the sections 'Design: Virtual Environment' and 'Psychometrical Results', these two conditions labelled High Presence and Low Presence differ in many other aspects than Presence, including in particular arousal, valence and monocular visual and auditory spatial cues. Thus, by contrasting the High Presence with the Low Presence condition, we cannot unambiguously infer brain regions specifically involved in presence. Nevertheless, we use the brain areas identified to be strongly activated in each particular contrast as masks for the following analyses (see below) in order to focus specifically on brain structures either activated or not activated by the High Presence condition. As expected, contrasting the High Presence condition with the Low Presence condition revealed both in adults and children widespread activation in brain areas known to be involved in egocentric spatial processing (dorsal visual stream, including superior and inferior parietal lobule and precuneus) (Gron et al., 2000; Jordan et al., 2004), object-based visual analyses and recognition (ventral visual stream, including fusiform gyrus, inferior and middle temporal gyrus) (Reddy and Kanwisher, 2006), sensory-motor processing (including gyrus postcentralis and premotor cortex), acoustic processing (auditory cortex) and emotion processing [including insula (Phillips et al., 2003); Figures S1A,B; Tables S2 and S3]. Comparing adults with children and children with adults in the contrast High Presence $>$ Low Presence revealed some small differences in the described functional systems (Tables S4 and S5). Overall, the above results clearly indicate that both age groups showed a highly similar brain activation pattern in the High Presence condition of this experimental paradigm.

\section{Correlational analyses of brain activation with Presence rating}

To investigate the neural basis of presence, we sought to uncover the brain activation pattern correlating with the subjective experience of presence. For this purpose, the difference in brain activation between the High and Low Presence condition was correlated with the difference in the presence rating between the same two presence conditions. Based on our a priori hypotheses, we focused our regression analyses on prefrontal brain regions, assuming that prefrontal brain regions (mainly in the dorsal and lateral part of the prefrontal cortex) (Koechlin et al., 2003) control the visual and auditory input of the realistic roller coaster ride and are thus involved in diminishing the presence experience. For the adults we found that the hemodynamic responses in two homologous prefrontal brain regions were negatively correlated with the subjective presence rating, namely the right and left dorsolateral prefrontal cortex (right DLPFC: $r=-0.467, p<0.001$; left DLPFC: $r=-0.408$, $p=0.003$; Figure $2 \mathrm{~A}$ ). The scatter plots and bar plots of Figures 2B,C show that the High Presence condition yielded a greater blood-oxygenation-level-dependent (BOLD) increment in the bilateral DLPFC in adults who reported a smaller amount of presence elevation.

In children, however, no negative correlation with any prefrontal brain region reached significance, even at a lowered threshold of $p<0.05$ (uncorrected). In order to examine whether children at least showed some tendencies for correlation in the DLPFC, we specified functional regions of interests (ROIs) in the bilateral DLPFC in children on the basis of the adult group analyses. These analyses revealed in children that the right but not left DLPFC showed a slight (but insignificant) negative correlation with the subjective experience of presence (Figure S2).

Finally, increased arousal and more positive valence ratings accompanying the High Presence condition in both groups (see above) cannot explain the presented correlational findings because regression analyses, with arousal and valence as a covariate, did not change in adults or children the observed correlational pattern between bilateral DLPFC and the subjective presence experience (Table S6).

\section{Voxel-based morphometry analyses}

The next step involved the use of voxel-based morphometry (VMB5) to examine whether the bilateral DLPFC activation observed in the correlational analyses showed structural differences between adults and children. Based on neuroanatomical 


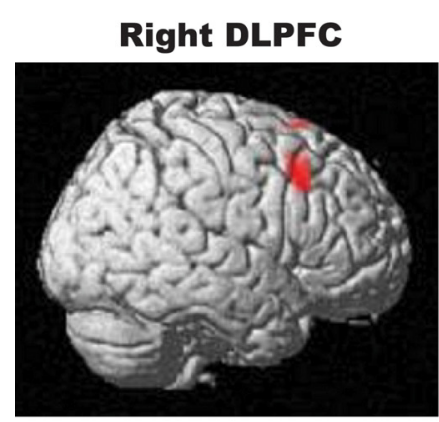

B
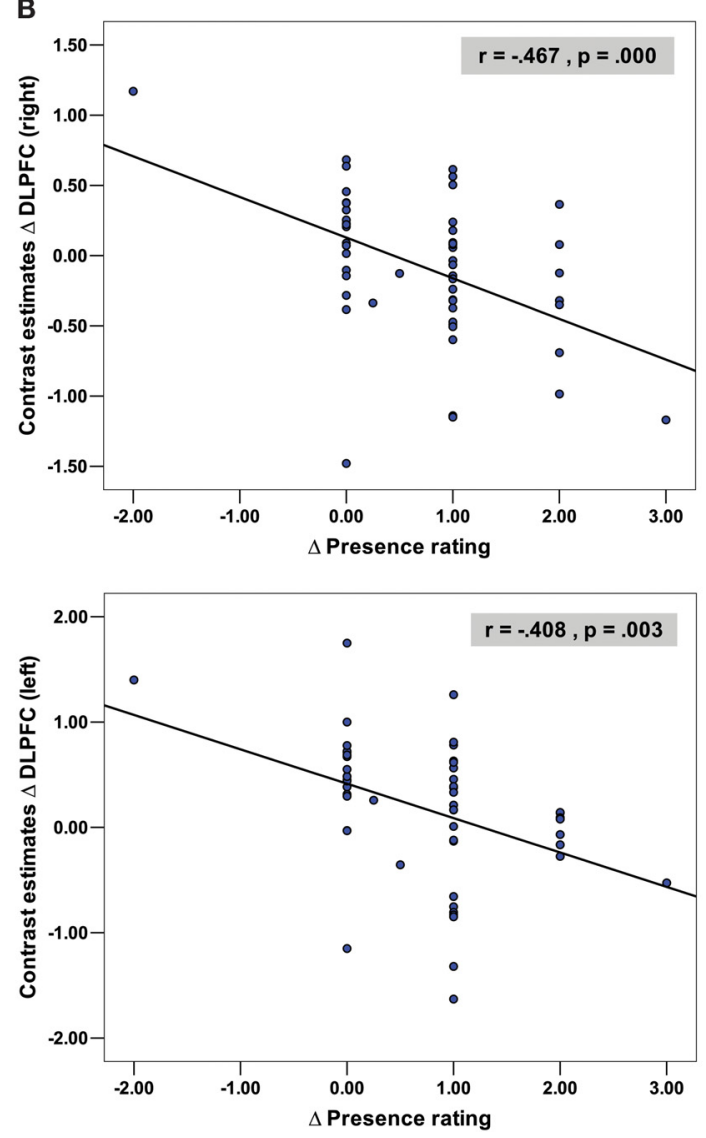

C
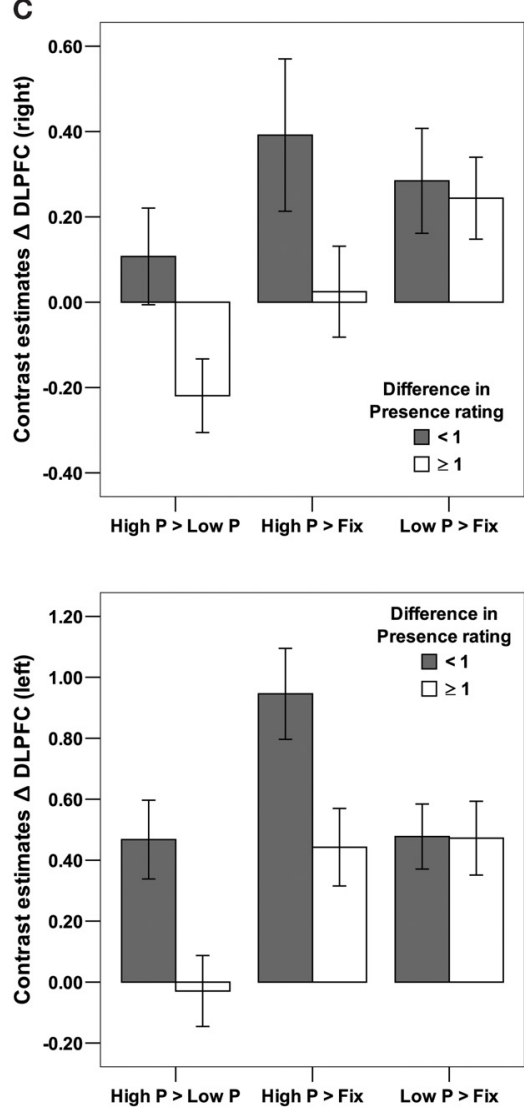

Figure 2 | Negative correlation of bilateral DLPFC with Presence experience in adults. (A) Depicted on the brain images are the bilateral DLPFC (right: $x=48, y=21, z=39$; BA = 9; left: $x=-54, y=15, z=36$, BA =9), which negatively correlated with the presence rating in adult subjects, indicating that the High Presence condition yielded a greater activation increase in the bilateral DLPFC in adult subjects who reported a smaller amount of Presence elevation between the High and Low Presence condition. (B) These negative correlations of DLPFC and presence ratings are depicted on the two scatter plots using functional ROIs. (C) In order to examine whether DLPFC activation differences in the High Presence (as expected), Low Presence or in both conditions contributed to the negative correlational pattern, we extracted contrast estimates difference in the bilateral DLPFC for the contrasts Low P $>$ Fix, High P $>$ Fix and High P > Low P (High P = High Presence, Low P = Low Presence and Fix = Fixation Baseline). Based on these contrast estimates, we created bar plots, broken down for adult subjects with difference in Presence rating $<$ or $\geq 1$, referred to as low and high Presence rating group, respectively. These bar plots illustrated that there is no difference in DLPFC activation between the two rating groups during the Low Presence condition (independent $t$-tests for right DLPFC: $t=0.26$, $\mathrm{df}=50, p=0.793$ and left DLPFC: $t=0.02, \mathrm{df}=50, p=0.977$ ). In contrast, during the High Presence condition, the low Presence rating group showed an increase, while the high Presence rating group showed no change (left DLPFC) or even a decrease in DLPFC (right DLPFC) activation, resulting in a significant group difference in the High Presence condition (independent $t$-tests for right DLPFC (one-tailed): $t=1.87, \mathrm{df}=50, p=0.033$ and left DLPFC: $t=2.54$, df $=50$, $p=0.014$ ). The rating groups significantly differed therefore in the contrast High $\mathrm{P}>$ Low $\mathrm{P}$ for the right (independent $t$-test: $t=2.32, \mathrm{df}=50, p=0.024$ ) and the left DLPFC (independent $t$-test: $t=2.80, \mathrm{df}=50, p=0.007$ ).

studies, it is known that the frontal cortex and the DLPFC in particular undergoes the longest period of time to mature, implying that the tissue itself continues to develop towards adult status well into adolescence (Giedd, 2004; Giedd et al., 1999; Gogtay et al., 2004; for a review see, Toga et al., 2006). In fact, we found in line with these studies that grey matter density and grey matter volume measures are increased in the bilateral DLPFC in children compared with adults, indicating that these prefrontal brain structures are not yet fully developed in children (Figure S3).

\section{Psychophysiological Interaction analyses (PPI)}

The bilateral DLPFC is strongly connected with many other regions of the brain (Amodio and Frith, 2006; Carmichael and Price, 1996; Croxson et al., 2005). We next focussed on this and whether we would find differences in effective connectivity between adults and children as well as between subjects experiencing strong and weak presence differences between the High and Low Presence conditions. For this purpose, we used Psychophysiological Interaction analyses (PPI) (Friston et al., 1997) to examine the task-dependent changes in effective connectivity between one brain area (in our case the right or left DLPFC) and other brain regions. In the context of this experiment, we are only interested in positive or negative connectivity of the left or right DLPFC with other regions of the brain, if and only if this connectivity is significantly stronger in the High Presence than in the Low Presence condition. Note that the word "down-regulation" will be synonymously used for negative connectivity, while the word "up-regulation" will be used for positive connectivity.

In our PPI analyses, we sought to answer two main questions. First, we were interested in whether DLPFC down-regulates activations in posterior brain regions found to be strongly activated in the High Presence compared with the Low Presence condition 
(depicted in Figure S1 and Tables S2 and S3). We investigated this connectivity because strong direct anatomical connections between DLPFC and posterior and in particular parietal regions have been demonstrated both in the macaque as well as human brain (Croxson et al., 2005). Second, we sought to determine whether DLPFC up-regulates and thus recruits additional brain regions which were not activated in the contrast High Presence $>$ Low Presence. In particular, we focused our analyses on the medial prefrontal cortex because lateral prefrontal brain regions have been shown to have strong anatomical connections with the medial prefrontal cortex (Amodio and Frith, 2006; Carmichael and Price, 1996), and because medial prefrontal cortex structures are functionally known to be involved in attention modulation, conflict monitoring and cognitive control (Botvinick et al., 1999; Carter et al., 1998; Ridderinkhof et al., 2004) as well as self-referential reflective activity (Amodio and Frith, 2006; D'Argembeau et al., 2005; Gusnard et al., 2001, for a review) We suggest that these processes might be involved in reducing the presence experience either in the context of critically evaluating the displayed virtual reality world or by directing attention away from the external virtual reality to internal self-reflective mental processes.

\section{PPI analyses, irrespective of the Presence rating, in adults and children}

In a first analysis, we attempted to answer the following questions: Do all adults use bilateral DLPFC to control and regulate their presence experience, irrespective of their presence rating? Moreover, are there lateralization differences between the left DLPFC and right DLPFC? We found that adults, irrespective of their presence rating, do indeed recruit bilateral DLPFC to modulate their presence experience. Moreover, there are strong lateralization differences. Whereas the right DLPFC mainly down-regulated the activation in the dorsal visual processing stream and in sensory-motor areas (Figures 3A,B and Table S7), the left DLPFC mainly up-regulated widespread areas of the medial prefrontal cortex, including anterior cingulate cortex (ACC, BA 24, 32, 25) and middle and superior frontal gyrus (BA $10,11)$. In addition, the left DLPFC showed positive connectivity with subcortical areas [including brainstem and mediodorsal thalamus which has prominent anatomical interconnections with the DLPFC (Giguere and Goldman-Rakic, 1988)] as well as areas of the primary and extrastriate visual system that were not activated in the contrast High Presence > Low Presence (Figures 3C,D and Table S7). Thus, the data suggest that in adult

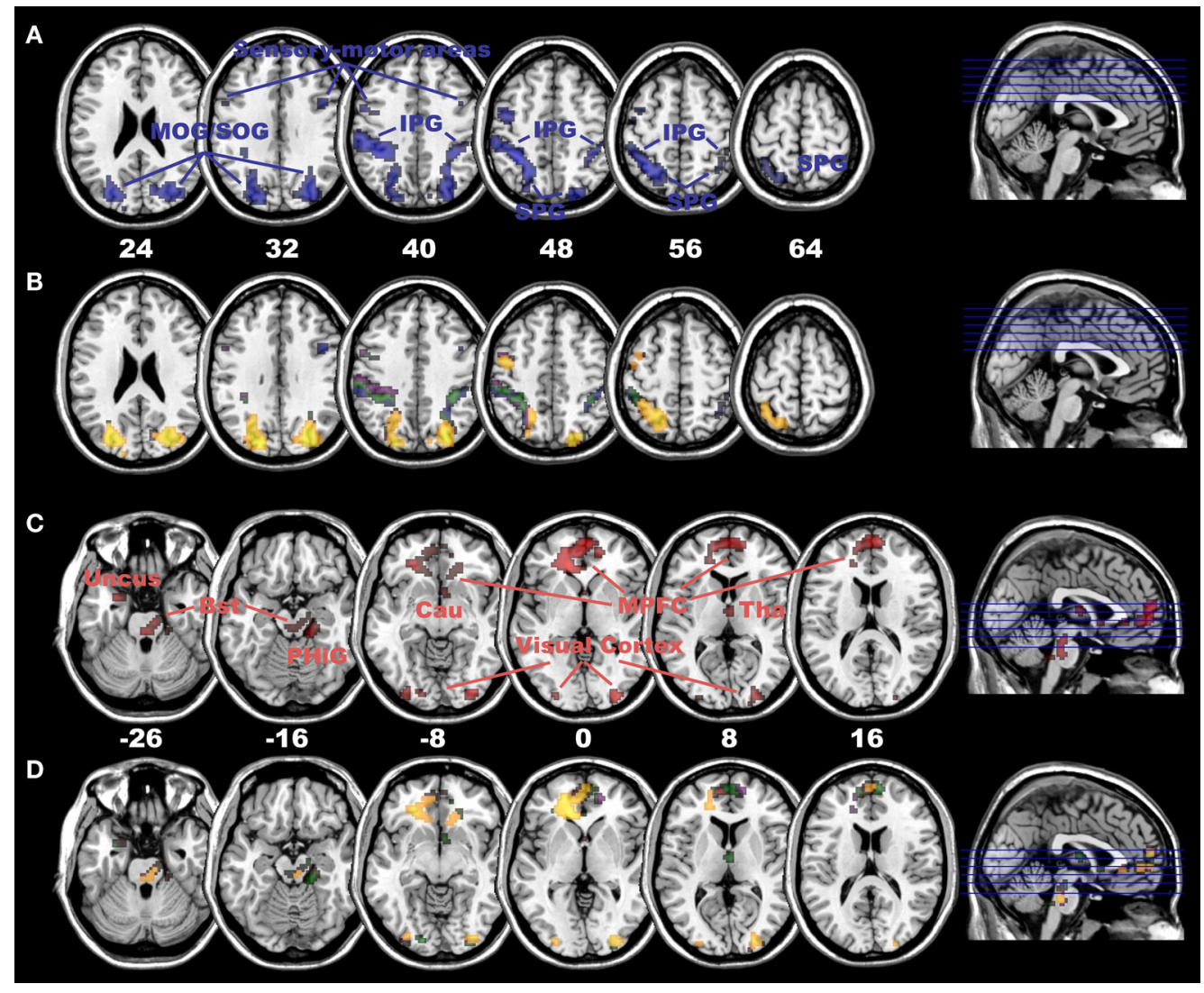

Figure 3 | Negative connectivity with right and positive connectivity with left DLPFC in adults. (A) Negative connectivity (blue colour) with right DLPFC in the dorsal visual stream (including superior and inferior parietal gyrus as well as superior occipital gyrus) and sensory-motor areas in adult subjects (at $p<0.005$, cluster extent: 10 voxels; SPG, superior parietal gyrus; IPG, inferior parietal gyrus; MOG, middle occipital gyrus; S0G, superior occipital gyrus). (B) Significant differences in negative connectivity between right DLPFC and left DLPFC in areas depicted in (A), at $p<0.005$ (yellow), $p<0.01$ (violet) and $p<0.05$ (green, all with a cluster extent of 10 voxels). Most areas, except for the ones in blue colour, depict a clear right-sided lateralization pattern in negative connectivity. (C) Positive connectivity (red colour) with left DLPFC in medial PFC (including ACC), extrastriate visual cortex and subcortical areas (including dorsomedial Thalamus and Brainstem) in adult subjects (at $p<0.005$, cluster extent: 10 voxels; MPFC, medial prefrontal cortex; Bst, Brainstem; Tha, Thalamus; Cau, Caudatus; PHiG, Parahippocampal Gyrus). (D) Significant differences in positive connectivity between left DLPFC and right DLPFC in areas depicted in (C), at $p<0.005$ (yellow), $p<0.01$ (violet) and $p<0.05$ (green, all with a cluster extent of 10 voxels, areas in red colour illustrate no significant difference). All areas, except for a few voxels in the medial PFC in red colour, depict a clear left-sided lateralization pattern in positive connectivity in adult subjects. 
subjects, the presence experience is mainly regulated and controlled by down-regulating the immersive visual-spatial input in posterior brain regions as well as by up-regulating medial areas of the PFC.

In the subsequent PPI analysis, we examined whether children demonstrated a connectivity pattern similar to that of adults, both irrespective of the presence rating and despite the fact that they showed comparatively smaller and a non-significant negative correlation with bilateral DLPFC and the presence rating (as has been shown above, see Figure S2). Interestingly, we found that children demonstrated a positive connectivity with their right DLPFC mainly in subcortical and emotional brain regions (including bilateral hippocampus/amygdala and insula) (Baumgartner et al., 2006a; Phillips et al., 2003), multisensory integration areas (temporo-parietal junction, BA 39, 40, 22) (Beauchamp et al., 2004; Downar et al., 2000), areas of the ventral visual stream (including fusiform gyrus and middle temporal gyrus, BA 37,21$)$ as well as two small clusters in the prefrontal cortex of which one was located in the medial PFC (Figures 4A,B and Table S8). Notably and in stark contrast to adult subjects, no negative connectivity with the right DLPFC and no positive connectivity with the left DLPFC were observed in the children, indicating a highly differential orchestration of the presence experience in adults and children in terms of lateralization pattern and connected brain regions.

The preceding analyses were conducted separately for children and adults and no direct statistical inference can be drawn from these analyses regarding group differences between adults and children. In order to elucidate the statistical age group differences in negative or positive connectivity, we thus directly compared the PPI results in adults with the PPI results in children and vice versa. Overall, these analyses confirmed most of the above reported descriptive differences between adults and children. Compared with children's connectivity with both the right and left DLPFC, we indeed found in adults, and irrespective of the presence rating, significantly stronger negative connectivity with their right DLPFC in many areas of the dorsal visual stream (including precuneus, inferior and superior parietal gyrus) and significantly stronger positive connectivity with their left DLPFC in the medial PFC [including ACC; Figure S4; all brain areas survive small volume ( $5 \mathrm{~mm}$ sphere) family-wise-error correction, see Table S9]. On the other hand, as compared with adult's connectivity with both their right and left DLPFC, the children demonstrated significantly stronger positive connectivity with their right DLPFC in the described subcortical and emotional brain areas, multisensory integration areas and areas of the ventral visual stream (Figure S5), again irrespective of the presence rating. Taken together, these statistical analyses confirmed, as hypothesized, that there are indeed age-group related differences in the orchestration of the experience of presence and that these differences are associated with those prefrontal brain structures that are very late to reach full maturity.

\section{PPI analyses of the two presence rating groups in adult and children subjects: Low Presence rating group > High Presence rating group}

So far, the analyses of the connectivity pattern of adults and children were performed irrespective of the presence rating, and the results suggest that all adults and children rather automatically engage prefrontal cortex structures to regulate and control the immersive experience of virtual reality. However, the correlational analyses revealed (mainly in adult subjects) that the bilateral DLPFC is more strongly activated in subjects who reported a reduced experience of presence. Thus, one would expect that this subgroup of subjects down-regulates or recruits additional brain structures to control the immersive visual and auditory spatial input. Compared with adult subjects of the High Presence rating group, we indeed found that the adult subjects of the Low Presence rating group down-regulated additional areas of the dorsal visual stream (including precuneus, superior and inferior

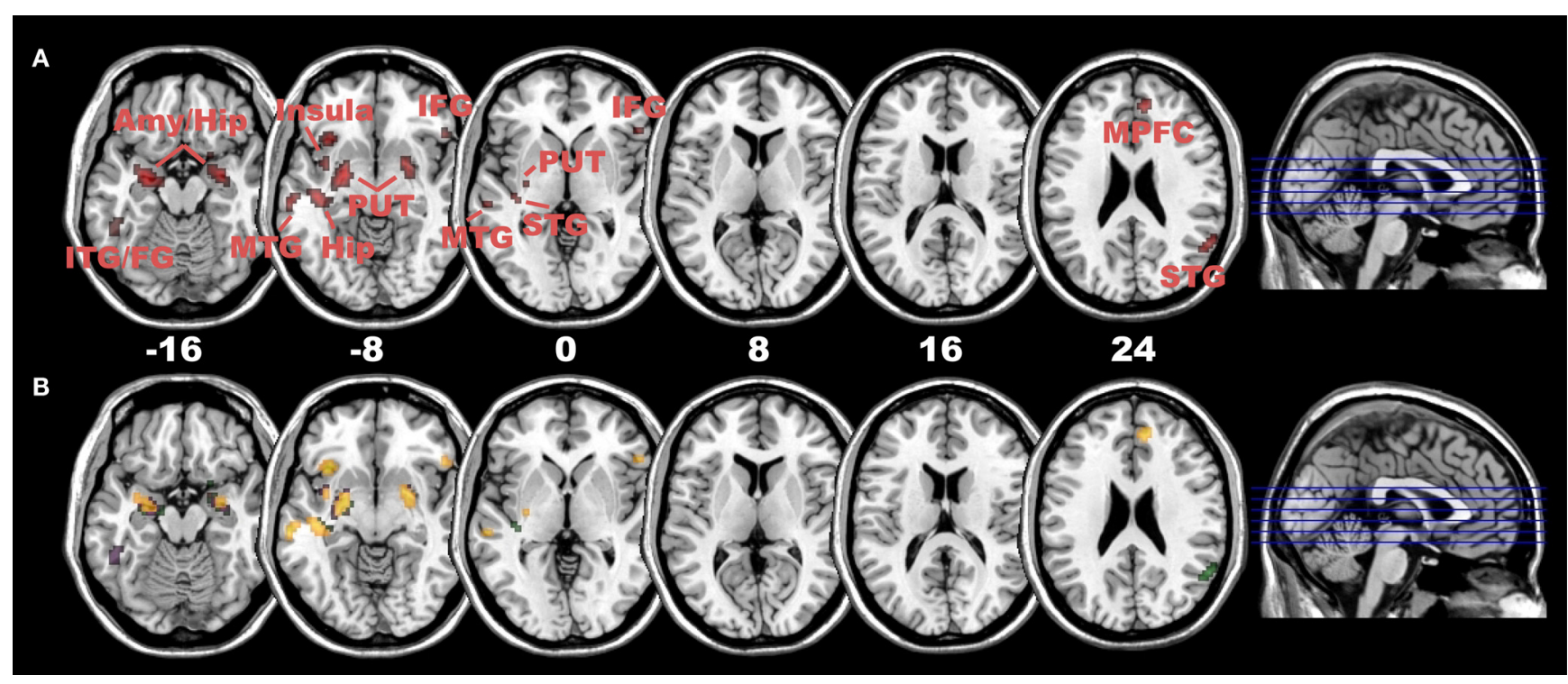

Figure 4 | Positive connectivity with right DLPFC in children. (A) Positive connectivity (red colour) with right DLPFC mainly in subcortical and emotional areas (including amygdala/hippocampus and insula) as well as multisensory integration areas (posterior STG), areas of the ventral visual processing stream (including Fusiform Gyrus) and small clusters in prefrontal areas (inferior and medial PFC) in children subjects (at $p<0.005$, cluster extent: 10 voxels; Amy, amygdala; Hip, hippocampus; ITG/MTG,STG, inferior/middle/superior temporal gyrus; Put, putamen; IFG, inferior frontal gyrus; MPFC, medial prefrontal cortex). (B) Significant differences in positive connectivity between right DLPFC and left DLPFC in areas depicted in (A), at $p<0.005$ (yellow), $p<0.01$ (violet) and $p<0.05$ (green, all with a cluster extent of 10 voxels). In children subjects, all areas depict a clear right-sided lateralization pattern in positive connectivity. 
parietal gyrus) with their right DLPFC and the posterior and ventral thalamus (pulvinar and sensory relay nuclei) with their bilateral DLPFC (Figures 5A,B and Table S10). In contrast, children of the Low Presence rating group showed no similar negative connectivity pattern (Figure 5C and for detailed statistical analyses Table S12), indicating that children of the Low Presence rating group use other strategies to control their presence experience. Indeed, we found that only children in the Low Presence rating group used their left DLPFC to down-regulate activation in the auditory cortex (BA 41/42) and temporal pole regions (Figure S6 and Table S11 and for detailed statistical analyses Table S12). Interestingly, we did not find in the adults or children of the Low
Presence rating group any additional recruitment of medial prefrontal or any other brain regions with their right or left DLPFC.

Taken together, these analyses suggest that adults and children of the Low Presence rating group use completely different strategies to regulate and control their presence experience. In the adults of the Low Presence rating group, suppression of presence experience involved the additional down-regulation of activation in the egocentric dorsal visual processing stream and in regions of the thalamus that relay sensory signals (visual and auditory) to the cerebral cortex (occipital, parietal and temporal) and play a role in gating and modulating attention toward or away from visual and auditory stimuli (Kastner et al., 2004;

A

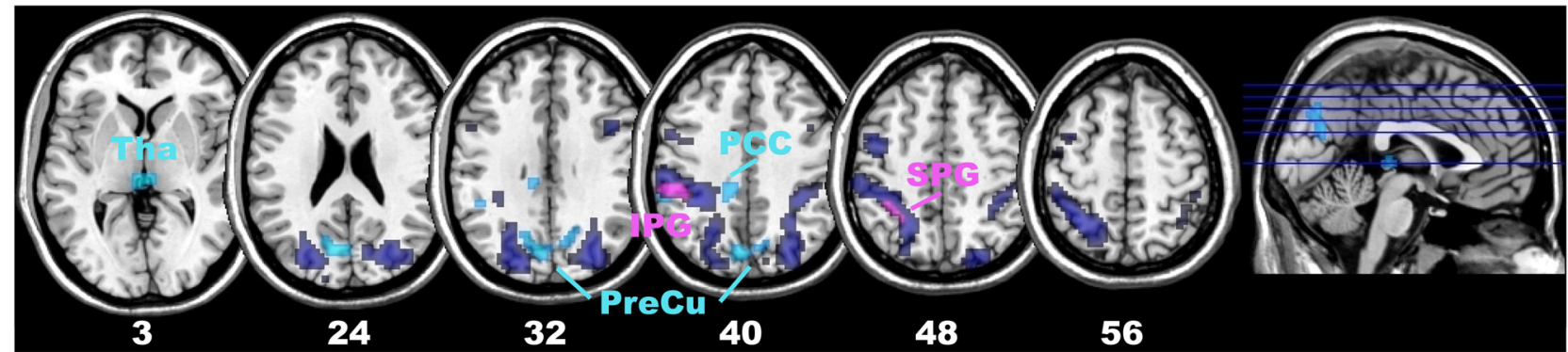

B

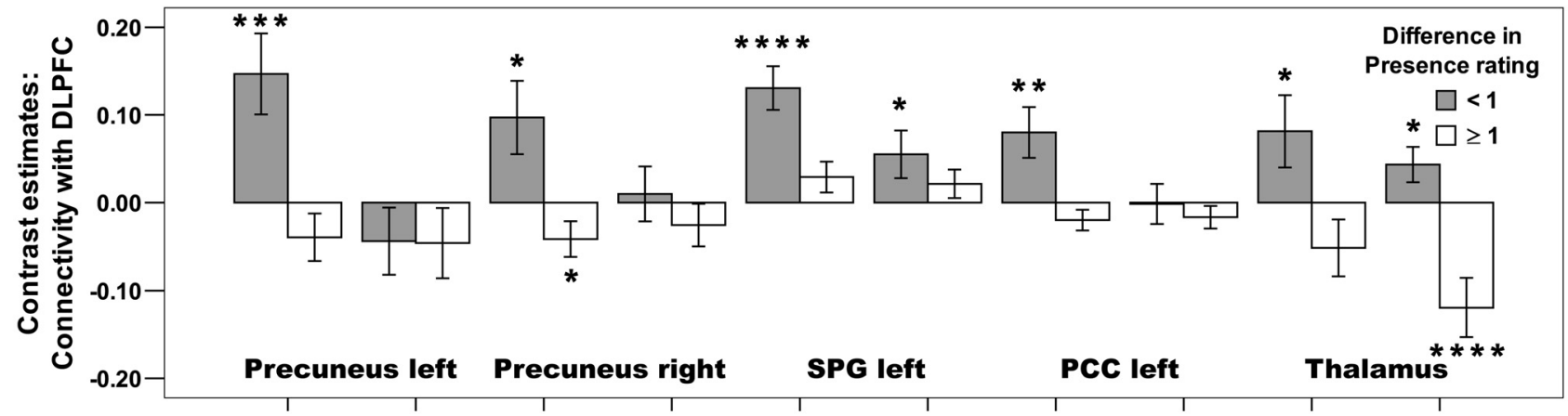

C

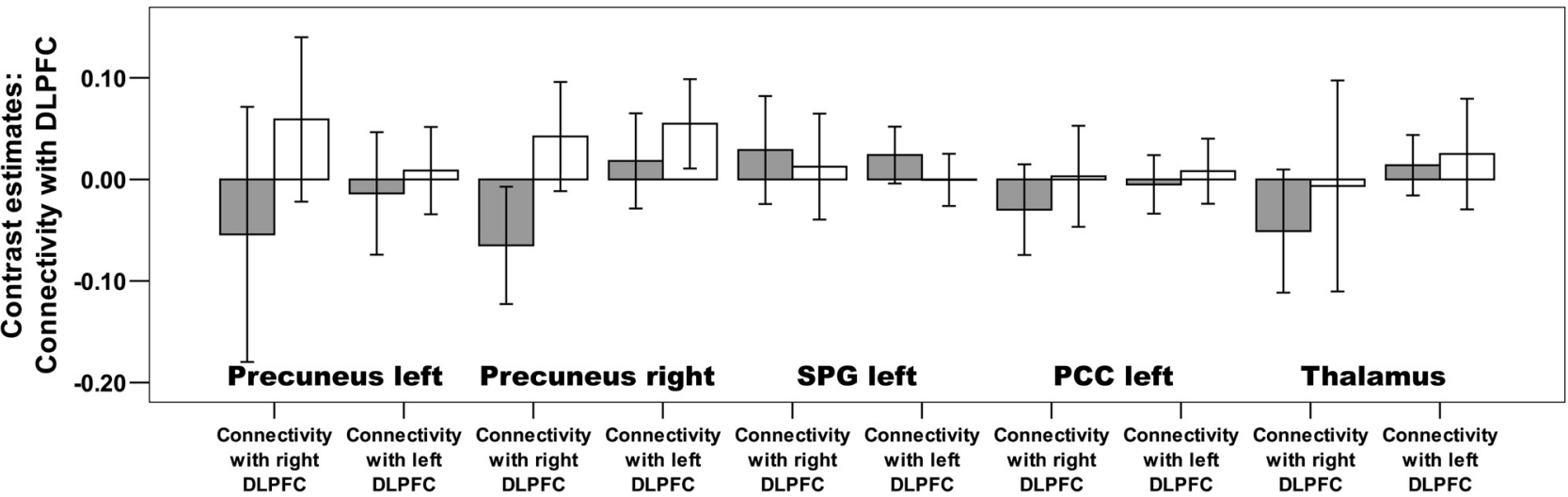

Figure 5 | Negative connectivity differences in adults: Low Presence rating group > High Presence rating group. (A) Whereas all adults, irrespective of their presence rating, used their right DLPFC to down-regulate activation in the dorsal visual stream and sensory-motor areas (depicted in dark blue colour, the same activation as in Figure $3 \mathrm{~A}$ ), subjects of the low Presence rating group used their right DLPFC to down-regulate additional areas of this dorsal visual system (including bilateral precuneus, inferior and superior parietal gyrus) as well as the posterior thalamus (depicted in light blue colour; $p<0.005$, cluster extent: 10 voxels; PreCu, Precuneus; IPG, inferior parietal gyrus; SPG, superior parietal gyrus; PCC, posterior cingulate cortex; Tha, Thalamus). Areas in violet colour are down-regulated by all adult subjects, but subjects of the low Presence rating group showed an even stronger down-regulation in this part of the inferior and superior parietal cortex. For those regions showing a differential group effect depicted in $(\boldsymbol{A})$, we also created regions of interests and extracted Beta estimates. Bar plots based on these Beta estimates are depicted for $(\boldsymbol{B})$ adults and $(\boldsymbol{C})$ children, broken down for the low (Difference in Presence rating $<1$ ) and high (Difference in Presence rating $\geq 1$ ) Presence rating groups. Positive values indicate negative connectivity, whereas negative values indicate positive connectivity. Asterisk indicate significant increase in negative or positive connectivity at $p<0.05(*), p \leq 0.01(* *), p \leq 0.005(* * *)$, or $p \leq 0.001$ (****). The bar plots in (B) illustrate that, except for the thalamus, an unilateral, right-sided and negative connectivity pattern has been observed in adult subjects of the low Presence rating group. In contrast, children of the low Presence rating group did not show this negative connectivity pattern in any brain region of the dorsal visual stream, either with the right or left DLPFC [depicted in (C), see Table S12 for detailed statistical analyses]. 
Kastner and Pinsk, 2004). In contrast, the children of the Low Presence rating group suppressed their presence experience by down-regulating the processing of the spatial auditory cues (delivered by the virtual reality software) in the auditory system and by down-regulating temporal pole regions known to be involved in emotion processing (Phillips et al., 2003).

\section{DISCUSSION}

The purpose of this investigation was to shed light on the potential impact of prefrontal brain structures and associated maturational changes on the experience of presence in an immersive VE. In view of the significant changes in the frontal cortex during the course of development to adulthood and of the role played by this brain area in exerting regulatory control over emotion and behaviour, we proposed that maturational differences in the PFC may be closely associated with differences between adults and children in orchestrating the experience of presence.

In fact, we found that prefrontal areas are strongly involved in modulating the experience of presence. But this applied only for adults, there being no such recruitment of these areas in children. The reason for this non-recruitment might lie in the relatively late maturation of the prefrontal cortex in children, a suggestion supported by our own anatomical data. We showed that two particular prefrontal cortical structures are not yet fully developed in children, as indicated by increased grey matter density and volume. Finally and most interesting, using Psychophysiological Interaction analyses (PPI), we demonstrated large differences in effective connectivity of these prefrontal brain structures between adults and children.

These effective connectivity analyses revealed in all adult subjects, and irrespective of presence rating, the specific recruitment of the right DLPFC to down-regulate the activation in the dorsal visual processing stream. Given the specific role played by the dorsal stream in egocentric processing of the visual environment, it is conceivable that the right DLPFC of adults is recruited as part of a strategy for regulating presence experience by constraining the egocentric processing of the roller coaster stimulus display. The pattern of effective connectivity in children stands in contrast to that of the adults. In children, the right DLPFC is involved in up-regulating activation in subcortical and emotional brain regions (including hippocampus, amygdala and insula), multi-sensory integration areas (temporo-parietal junction), and areas of the ventral visual processing stream. In processing ongoing VE stimuli, this possibly reflects a distinctly different strategy to that of adults, given the fact that most of the modulated brain regions, including in particular the bilateral hippocampus and the areas of the ventral visual stream, are part of a neurofunctional network involved in allocentric, object-based spatial processing, which is independent of the observer's spatial location (Jordan et al., 2004; Nadel and Hardt, 2004; Zaehle et al., 2007). We propose that the up-regulation of an allocentric spatial reference frame interferes with the experience of presence under stimulus conditions specifically designed to facilitate presence by inducing egocentric processing of displayed spatial information. In contrast to the adults' direct strategy, up-regulation of allocentric spatial processing by children may reflect an indirect approach in modulating the experience of presence. We speculate that this indirect approach is most likely less efficient than the direct approach applied by adults. This speculation is supported by the finding that the indirect strategy involves in addition to the up-regulation of the allocentric spatial reference frame a concurrent activation of areas associated with affective processing, including bilateral amygdala and insula. The activation of these areas during arousing VE experience strongly suggests that the children are more susceptible to the arousing impact of the visual and auditory spatial stimuli and are thus less able to regulate and control the experience of presence during arousing VE.

In addition to the regulation of the egocentric spatial processing in posterior brain regions, all adult subjects, irrespective of their subjective presence experience, used an additional regulation strategy. Their left DLPFC recruited widespread areas of the medial PFC (including ACC) that are together known to be functionally involved in attention modulation, conflict monitoring, cognitive control (Botvinick et al., 1999; Carter et al., 1998; Ridderinkhof et al., 2004) and self-referential reflective activity (Amodio and Frith, 2006; D'Argembeau et al., 2005; Gusnard et al., 2001, for a review). Moreover, these brain regions of the medial prefrontal cortex are known to be part of a "default mode of brain function" whose activity is ongoing during rest and suspended during performance of externally cued tasks (Greicius et al., 2003, 2008; Gusnard et al., 2001; Rilling et al., 2007). Taken together, this recruitment of medial prefrontal regions might indicate that adults are strongly using internal self-reflective control processes while they are exposed to VE stimuli. Or in other words, adults appear to control and regulate their presence experience by critically evaluating and monitoring the presented VE stimuli, and/or by directing attention away from the external virtual reality to internal self-reflective mental processes. Children on the other hand did not, or at least to a greatly reduced extent, apply these "strategies". Thus, children do not rely on this control process of the medial PFC - most likely because of the not fully matured prefrontal cortex (Giedd, 2004; Giedd et al., 1999; Gogtay et al., 2004). This is also consistent with many studies demonstrating delayed maturation of several executive functions in children (Romine and Reynolds, 2005; Segalowitz and Davies, 2004).

Taken together, this is the first study to indicate that adults use two major strategies to modulate the experience of presence and to show that these strategies are completely absent in children. These strategies are the down-regulation of the egocentric visual processing in posterior parietal brain regions using the right $D L P F C$ and the up-regulation of medial PFC structures using the left DLPFC. This leads inevitably to the question which of these two strategies is the more important for regulating the experience of presence. The evidence from our own study indicates that the control of the egocentric view with the right DLPFC might be more critical. This is indicated by the fact that the adult subjects who experienced weak presence (the Low Presence rating group) compared with those experiencing strong presence (the High Presence rating group) showed an additional downregulation associated with the right DLPFC in brain regions coding for this egocentric view. Notably, no additional recruitment of medial PFC or any other brain regions with the right or the left DLPFC were observed. The complete absence of any additional recruitment of medial PFC structures may be taken as to suggest that the down-regulation of the egocentric dorsal visual processing stream with the right DLPFC is the more important and efficient strategy in controlling and regulating the presence experience. In line with this interpretation, both "out-of body" phenomena and the "rubber-hand illusions" also point to posterior parietal brain regions as well as multi-sensory premotor regions as playing an important role in modulating 
illusory body phenomena (Blanke, 2004; Blanke et al., 2004; Ehrsson, 2007; Ehrsson et al., 2004, 2007; Lenggenhager et al., 2007). It is noteworthy that the right DLPFC was also found in our study to control the activation in the bilateral multi-sensory premotor cortex and as a consequence, to modulate the presence experience.

In summary, this study is the first to (1) reveal the involvement of the right and to a lesser extent of the left DLPFC as highly specific neural correlates for the orchestration of the presence experience in adults, and to (2) demonstrate the striking absence of these adult-like mechanisms in children. These findings highlight the relative susceptibility of children to the experience of presence in simulated environments and may have important consequences for pedagogical and educational interventions. One consequence might be that one should be more reluctant to expose children to emotional virtual stimuli as currently practiced.

\section{CONFLICT OF INTEREST STATEMENT}

The authors declare that the research was conducted in the absence of any commercial of financial relationships that could be construed as a potential conflict of interests.

\section{ACKNOWLEDGEMENTS}

This work is funded under the European Union FET project PRESENCCIA (Contract Number 27731) and the National Competence Center for Research (NCCR) in Affective Sciences. The NCCR is financed by the Swiss National Science Foundation.

\section{SUPPLEMENTARY MATERIAL}

The Supplementary Material for this article can be found online at http://www.frontiersin.org/humanneuroscience/paper/10.3389/ neuro.09/008.2008/.

\section{REFERENCES}

Amodio, D. M., and Frith, C. D. (2006). Meeting of the minds: the medial frontal cortex and social cognition. Nat. Rev. Neurosci. 7, 269-277.

Annett, M. (1970). A classification of hand preference by association analysis. Br. J. Clin. Psychol. 61, 303-321.

Aron, A. R., Robbins, T. W., and Poldrack, R. A. (2004). Inhibition and the right inferior frontal cortex. Trends Cogn. Sci. 8(4), 170-177.

Ashburner, J., and Friston, K. J. (2000). Voxel-based morphometry - the methods. Neuroimage 11(6 Pt 1), 805-821.

Ashburner, J., and Friston, K. J. (2005). Unified segmentation. Neuroimage 26(3), 839-851.

Baumgartner, T., Lutz, K., Schmidt, C. F., and Jancke, L. (2006a). The emotional power of music: how music enhances the feeling of affective pictures. Brain Res. 1075(1), 151-164.

Baumgartner, T., Valko, L., Esslen, M., and Jancke, L. (2006b). Neural correlate of spatial presence in an arousing and noninteractive virtual reality: an EEG and psychophysiology study. Cyberpsychol. Behav. 9(1), 30-45.

Beauchamp, M. S., Lee, K. E., Argall, B. D., and Martin, A. (2004). Integration of auditory and visual information about objects in superior temporal sulcus. Neuron 41(5), 809-823.

Blanke, O. (2004). Out of body experiences and their neural basis. BMJ 329(7480), 1414-1415.

Blanke, O., Landis, T., Spinelli, L., and Seeck, M. (2004). Out-of-body experience and autoscopy of neurological origin. Brain 127(Pt 2), 243-258

Botvinick, M., Nystrom, L. E., Fissell, K., Carter, C. S., and Cohen, J. D. (1999). Conflict monitoring versus selection-for-action in anterior cingulate cortex. Nature 402(6758), 179-181.

Burgund, E. D., Kang, H. C., Kelly, J. E., Buckner, R. L., Snyder, A. Z. et al. (2002). The feasibility of a common stereotactic space for children and adults in fMRI studies of development. Neuroimage 17(1), 184-200.

Carmichael, S. T., and Price, J. L. (1996). Connectional networks within the orbital and medial prefrontal cortex of macaque monkeys. J. Comp. Neurol. 371(2), 179-207.
Carter, C. S., Braver, T. S., Barch, D. M., Botvinick, M. M., Noll, D. et al. (1998). Anterior cingulate cortex, error detection, and the online monitoring of performance. Science 280(5364), 747-749.

Croxson, P. L., Johansen-Berg, H., Behrens, T. E., Robson, M. D., Pinsk, M. A et al. (2005). Quantitative investigation of connections of the prefrontal cortex in the human and macaque using probabilistic diffusion tractography. J. Neurosci. 25(39), 8854-8866.

D’Argembeau, A., Collette, F., Van der Linden, M., Laureys, S., Del Fiore, G. et al. (2005). Self-referential reflective activity and its relationship with rest: a PET study. Neuroimage 25(2), 616-624.

Downar, J., Crawley, A. P., Mikulis, D. J., and Davis, K. D. (2000). A multimodal cortical network for the detection of changes in the sensory environment. Nat. Neurosci. 3(3), 277-283.

Ehrsson, H. H. (2007). The experimental induction of out-of-body experiences. Science 317(5841), 1048.

Ehrsson, H. H., Spence, C., and Passingham, R. E. (2004). That's my hand! Activity in premotor cortex reflects feeling of ownership of a limb. Science 305(5685), 875-877.

Ehrsson, H. H., Wiech, K., Weiskopf, N., Dolan, R. J., and Passingham, R. E. (2007). Threatening a rubber hand that you feel is yours elicits a cortical anxiety response. Proc. Natl. Acad. Sci. U.S.A. 104(23), 9828-9833.

Eisenberger, N. I., Taylor, S. E., Gable, S. L., Hilmert, C. J., and Lieberman, M. D. (2007). Neural pathways link social support to attenuated neuroendocrine stress responses. Neuroimage 35(4), 1601-1612.

Forman, S. D., Cohen, J. D., Fitzgerald, M., Eddy, W. F., Mintun, M. A. et al. (1995). Improved assessment of significant activation in functional magnetic resonance imaging (fMRI): use of a cluster-size threshold. Magn. Reson. Med. 33(5), 636-647.

Freeman, J., Avons, S. E., Pearson, D. E., and Ijsselsteijn, W. A. (1999). Effects of sensory information and prior experience on direct subjective ratings of presence. Presence: Teleop. Virt. 8, 1-13.

Friston, K. J., Buechel, C., Fink, G. R., Morris, J., Rolls, E. et al. (1997) Psychophysiological and modulatory interactions in neuroimaging. Neuroimage 6(3), 218-229.

Garavan, H., Ross, T. J., and Stein, E. A. (1999). Right hemispheric dominance of inhibitory control: an event-related functional MRI study. Proc. Natl. Acad. Sci. U.S.A. 96(14), 8301-8306.

Giedd, J. N. (2004). Structural magnetic resonance imaging of the adolescent brain. Ann. N.Y. Acad. Sci. 1021, 77-85.

Giedd, J. N., Blumenthal, J., Jeffries, N. O., Castellanos, F. X., Liu, H. et al. (1999). Brain development during childhood and adolescence: a longitudinal MRI study. Nat. Neurosci. 2(10), 861-863.

Giguere, M., and Goldman-Rakic, P. S. (1988). Mediodorsal nucleus: areal, laminar, and tangential distribution of afferents and efferents in the frontal lobe of rhesus monkeys. J. Comp. Neurol. 277(2), 195-213.

Gogtay, N., Giedd, J. N., Lusk, L., Hayashi, K. M., Greenstein, D. et al. (2004). Dynamic mapping of human cortical development during childhood through early adulthood. Proc. Natl. Acad. Sci. U.S.A. 101(21), 8174-8179.

Greicius, M. D., Krasnow, B., Reiss, A. L., and Menon, V. (2003). Functional connectivity in the resting brain: a network analysis of the default mode hypothesis. Proc. Natl. Acad. Sci. U.S.A. 100(1), 253-258.

Greicius, M. D., Supekar, K., Menon, V., and Dougherty R. F. (2008). Resting-state functional connectivity reflects structural connectivity in the default mode network. Cereb Cortex.

Gron, G., Wunderlich, A. P., Spitzer, M., Tomczak, R., and Riepe, M. W. (2000). Brain activation during human navigation: gender-different neural networks as substrate of performance. Nat. Neurosci. 3(4), 404-408.

Gusnard, D. A., Akbudak, E., Shulman, G. L., and Raichle, M. E. (2001). Medial prefrontal cortex and self-referential mental activity: relation to a default mode of brain function. Proc. Natl. Acad. Sci. U.S.A. 98(7), 4259-4264.

Harbaugh, W. T., Mayr, U., and Burghart, D. R. (2007). Neural responses to taxation and voluntary giving reveal motives for charitable donations. Science 316, 1622-1625.

Ijsselsteijn, W. A., de Ridder, H., Freeman, J., Avons, S. E., and Bouwhuis, D. (2001). Effects of stereoscopic presentation, image motion, and screen size on subjective and objective corroborative measures of presence. Presence 10(3), 298-311.

Ijsselsteijn, W. A., de Ridder, H., Hamberg, R., Bouwhuis, D., and Freeman, J. (1998). Perceived depth and the feeling of presence in 3DTV. Displays 18, 207-214.

Ijsselsteijn, W. A., and Riva, G. (2003). Being there: the experience of presence in mediated environments. In Being There: Concepts, Effects, and Measurements of User Presence in Synthetic Environments, G. Riva, F. Davide, and W. A. Ijsselsteijn, eds (Amsterdam, Ios Press).

Jordan, K., Schadow, J., Wuestenberg, T., Heinze, H. J., and Jancke, L. (2004). Different cortical activations for subjects using allocentric or egocentric strategies in a virtual navigation task. Neuroreport 15(1), 135-140.

Kang, H. C., Burgund, E. D., Lugar, H. M., Petersen, S. E., and Schlaggar, B. L. (2003). Comparison of functional activation foci in children and adults using a common stereotactic space. Neuroimage 19(1), 16-28. 
Kastner, S., O'Connor, D. H., Fukui, M. M., Fehd, H. M., Herwig, U. et al. (2004) Functional imaging of the human lateral geniculate nucleus and pulvinar J. Neurophysiol. 91(1), 438-448.

Kastner, S., and Pinsk, M. A. (2004). Visual attention as a multilevel selection process. Cogn. Affect. Behav. Neurosci. 4(4), 483-500.

Klinger, E., Bouchard, S., Legeron, P., Roy, S., Lauer, F. et al. (2005). Virtual reality therapy versus cognitive behavior therapy for social phobia: a preliminary controlled study. Cyberpsychol. Behav. 8(1), 76-88.

Klinger, E., Chemin, I., Lebreton, S., and Marie, R. M. (2006). Virtual action planning in Parkinson's disease: a control study. Cyberpsychol. Behav. 9(3), 342-347.

Knoch, D., Gianotti, L. R. R., Pascual-Leone, A., Treyer, V., Regard, M. et al. (2006a). Disruption of right prefrontal cortex by low-frequency repetitive transcranial magnetic stimulation induces risk-taking behavior. J. Neurosci. 26(24), 6469-6472.

Knoch, D., Pascual-Leone, A., Meyer, K., Treyer, V., and Fehr, E. (2006b). Diminishing reciprocal fairness by disrupting the right prefrontal cortex. Science 314(5800), 829-832.

Koechlin, E., and Hyafil, A. (2007). Anterior prefrontal function and the limits of human decision-making. Science 318(5850), 594-598.

Koechlin, E., Ody, C., and Kouneiher, F. (2003). The architecture of cognitive control in the human prefrontal cortex. Science 302(5648), 1181-1185.

Lang, P. J. (1985). The Cognitive Psychophysiology of Emotion: Anxiety and the Anxiety Disorders. Hillsdale, NJ, Lawrence Erlbaum.

Lenggenhager, B., Tadi, T., Metzinger, T., and Blanke, O. (2007). Video ergo sum manipulating bodily self-consciousness. Science 317(5841), 1096-1099.

Nadel, L., and Hardt, O. (2004). The spatial brain. Neuropsychology 18(3), 473-476.

Parsons, T. D., Bowerly, T., Buckwalter, J. G., and Rizzo, A. A. (2007). A controlled clinical comparison of attention performance in children with ADHD in a virtual reality classroom compared to standard neuropsychological methods. Child Neuropsychol. 13(4), 363-381.

Parsons, T. D., and Rizzo, A. A. (2008). Affective outcomes of virtual reality exposure therapy for anxiety and specific phobias: a meta-analysis. J. Behav Ther. Exp. Psychiatry 39, 250-261.

Phillips, M. L., Drevets, W. C., Rauch, S. L., and Lane, R. (2003). Neurobiology of emotion perception I: the neural basis of normal emotion perception. Biol. Psychiatry 54(5), 504-514.

Reddy, L., and Kanwisher, N. (2006). Coding of visual objects in the ventral stream. Curr. Opin. Neurobiol. 16(4), 408-414.
Ridderinkhof, K. R., Ullsperger, M., Crone, E. A., and Nieuwenhuis, S. (2004). The role of the medial frontal cortex in cognitive control. Science 306(5695), 443-447.

Rilling, J. K., Barks, S. K., Parr, L. A., Preuss, T. M., Faber, T. L. et al. (2007) A comparison of resting-state brain activity in humans and chimpanzees. Proc. Natl. Acad. Sci. U.S.A. 104(43), 17146-17151.

Romine, C. B., and Reynolds, C. R. (2005). A model of the development of frontal lobe functioning: findings from a meta-analysis. Appl. Neuropsychol. 12(4), 190-201.

Sanchez-Vives, M. V., and Slater, M. (2005). From presence to consciousness through virtual reality. Nat. Rev. Neurosci. 6(4), 332-339.

Schaik, P., Turnbull, T., Wersch, A., and Drummond, S. (2004). Presence within a mixed reality environment. Cyberpsychol. Behav. 7(5), 540-552.

Segalowitz, S. J., and Davies, P. L. (2004). Charting the maturation of the frontal lobe: an electrophysiological strategy. Brain Cogn. 55(1), 116-133.

Slater, M., Pertaub, D. P., Barker, C., and Clark, D. M. (2006). An experimental study on fear of public speaking using a virtual environment. Cyberpsychol. Behav. 9(5), 627-633.

Sowell, E. R., Thompson, P. M., Holmes, C. J., Batth, R., Jernigan, T. L. et al (1999). Localizing age-related changes in brain structure between childhood and adolescence using statistical parametric mapping. Neuroimage 9(6 Pt 1), 587-597.

Steuer, J., Biocca, F., and Levy, M. R. (1995). Defining virtual reality: dimensions determining telepresence. In Communication in the Age of Virtual Reality, F. Biocca and M. R. Levy, eds (Hillsdale, NJ, Lawrence Erlbaum Associates), pp. 33-56.

Thielscher, A., and Pessoa, L. (2007). Neural correlates of perceptual choice and decision making during fear-disgust discrimination. J. Neurosci. 27(11) 2908-2917.

Toga, A. W., Thompson, P. M., and Sowell, E. R. (2006). Mapping brain maturation. Trends Neurosci 29(3), 148-159.

Vorderer, P., Wirth, W., Saari, T., Baumgartner, T., Jancke, L. et al. (2004). Development of the MEC spatial presence questionnaire (MEC SPQ). Report to the European Community, Project Presence, MEC

Wirth, W., Hartmann, T., Böcking, S., Vorderer, P., Klimmt, C. et al. (2007). A process model of the formation of spatial presence experiences. Media Psychol 9(3), 493-525.

Zaehle, T., Jordan, K., Wustenberg, T., Baudewig, J., Dechent, P. et al. (2007). The neural basis of the egocentric and allocentric spatial frame of reference. Brain Res 1137(1), 92-103. 